\title{
Abdominal Bloating and Distension: What Is the Role of the Microbiota
}

\author{
B. Issa $\cdot$ N. A. Wafaei $\cdot$ P. J. Whorwell
}

Received: 12 April 2011 / Accepted: 12 July 2011/Published online: 29 July 2011

(C) Springer Science+Business Media, LLC 2011

\begin{abstract}
Most patients with irritable bowel syndrome complain of a sensation of an increase in pressure within their abdomen during the course of the day which is called bloating and, in approximately half of these individuals, this symptom is accompanied by an actual increase in abdominal girth, which is referred to as distension. The pathophysiology of these two phenomena is somewhat different and it is now recognised that a whole variety of overlapping mechanisms are involved. Some of these are potentially amenable to treatment by modification of the bacterial flora of the gut and this article reviews the evidence for this.
\end{abstract}

Keywords Irritable bowel syndrome $\cdot$ Bloating ·

Distension $\cdot$ Microbiome

\section{Background}

Patients with functional gastrointestinal disorders, especially irritable bowel syndrome (IBS), frequently complain of bloating and distension and it is not uncommon for them

B. Issa · P. J. Whorwell

Neurogastroenterology Unit, Department of Translational

Medicine, University of Manchester, Manchester, UK

N. A. Wafaei

Department of General and Colorectal Surgery,

Fairfield General Hospital, The Pennine Acute Hospitals NHS

Trust, Bury BL9 7TD, UK

P. J. Whorwell ( $\square)$

Education and Research Centre, Wythenshawe Hospital,

Manchester M23 9LT, UK

e-mail: Peter.whorwell@uhsm.nhs.uk to report that these features often occur on a daily basis and, as a consequence, are especially bothersome. Another problem is that their management has always proved challenging, probably because, until recently, their pathophysiology has been poorly understood. However, the situation is now beginning to change with the recognition that a whole variety of factors may be involved and, importantly, that bloating and distension should be regarded as different but overlapping conditions. It is now suggested that the term bloating should be applied to the sensation of an increased pressure within the abdomen and that the term distension should only be used when this sensation is accompanied by an actual increase in abdominal girth. Recent research has indicated that in approximately $50 \%$ of patients reporting bloating, this sensation is accompanied by distension [1] and that there are subtle differences in the mechanisms underlying these conditions $[2,3]$.

\section{Putative Causes of Bloating and Distension}

Research on bloating and distension has been hampered by the lack of availability of suitable methods of investigation but this has been changed by the advent of techniques such as the gas challenge technique [4,5], abdominal inductance plethysmography [6,7], abdominal and diaphragmatic electromyography [8, 9], CT scanning [10], and no doubt magnetic resonance imaging will contribute in the future. The application of such methodology has resulted in the recognition that bloating tends to be associated with a phenotype characterised by increased visceral sensitivity [11], impaired gas handling [4], and diarrhoea [12], whereas distension is more often related to constipation [12], delayed gastrointestinal transit [12], weak abdominal musculature [13], and an abnormal accommodation reflex 
[8-10] where there is paradoxical contraction of diaphragmatic and abdominal muscles in response to an increase in abdominal pressure. In addition, there is evidence that in at least a proportion of patients with IBS, the bacterial flora of the gut may be disturbed [14-16] and additionally, fermentation may also contribute to symptoms [17] raising the possibility that these two factors may also contribute to both bloating and distension.

\section{Treatment Approaches to Bloating and Distension}

As a consequence of this evidence that bloating and distension differ mechanistically to some extent, it follows that the success of treatment is likely to be enhanced if the appropriate pathophysiological abnormality is targeted in a particular individual. For example, relieving constipation is more likely to improve distension whereas for a patient with bloating alone, aiming to reduce visceral hypersensitivity is a more logical strategy. Unfortunately, the situation is somewhat complicated by the fact that, at least in the UK, patients tend to describe their problem as bloating, irrespective of whether they are troubled by bloating or distension, but at least these pathophysiological observations provide a framework for a systematic approach to managing these problems.

Of the various pathophysiological mechanisms involved in bloating and distension that have been enumerated above, it seems reasonable to speculate that dysbiosis, gas handling, constipation, diarrhoea, visceral hypersensitivity, and fermentation might be amenable to modification by use of probiotics or antibiotics and there follows a discussion providing some evidence to support this view.

\section{The Therapeutic Potential of Antibiotics and Probiotics}

The notion that the microbiota of the gastrointestinal tract may be disturbed in patients with IBS has largely been prompted by two observations. First, for some patients with IBS there seems to be evidence of a low-grade inflammatory response within their gastrointestinal mucosa. Second, for others there is evidence of small intestinal bacterial over-growth (SIBO). Given that some probiotic bacteria have been shown to have a range of anti-inflammatory activity it is not surprising that their use has been advocated as a possible treatment for IBS. A relatively large number of controlled trials have been reported to date and, although their design has been rather variable, most have shown some evidence of a positive effect [18]. However, it should also be noted that different symptoms seem to respond to different probiotics but in a significant proportion of studies an improvement in subjectively reported bloating has been documented. Evidence for SIBO has come from studies using breath testing techniques after carbohydrate ingestion and, depending on the substrate used, the reported prevalence of the SIBO in IBS is somewhat variable [19]. Nevertheless, these observations have led some researchers to speculate that antibiotics, especially if they are of the non-absorbable variety, might have therapeutic potential in IBS, and the first studies used neomycin with positive results [20]. Subsequently, rifaximin has become the antibiotic of choice and there is now evidence that the use of this drug over a period of 7-14 days can reduce symptoms, including gaseousness and bloating, not only in the short term but also for a period of up to 3 months [21-23]. Obviously, the prolonged use of antibiotics to treat a condition such as IBS could not be advocated, but if a short course of a non-absorbable antibiotic is beneficial with a substantial carry over effect, then this option might well have some utility. One of the problems with any research in this area has been that, until recently, evidence of the role of bacteria in conditions such as IBS has been hampered by the fact that it could only be investigated indirectly by the use of techniques such as breath testing, or directly by culture techniques which are impeded by the fact that only a relatively small proportion of the gut microbiota can be cultured [19, 24, 25]. However, with the advent of molecular techniques, it is now possible to more accurately address this issue, and, after use of such approaches, reports are already emerging of variations in the microbiological profiles of gut bacteria in IBS [16, 26-32].

\section{Specific Evidence of the Benefit of Individual Antibiotics or Probiotics in Bloating and Distension}

Over the years, the Barcelona group have undertaken a series of elegant studies showing that compared with controls, patients with IBS have impaired handling of a gas load leading to gas trapping and the symptom of bloating [4]. This observation suggests that first, patients with bloating should avoid carbonated drinks and foods that tend to produce gas, and, second, that an attempt to alter endogenous gas production by modification of the bacterial flora is worthy of consideration, and that this could possibly be achieved by the use of an antibiotic or a probiotic. Certainly, some of the studies using rifaximin (Fig. 1) have supported this view $[21-23,33]$ and it is noteworthy that a reduction in bloating has been reported after use of some probiotics, for example VSL\#3 [34, 35] and Bifidobacterium infantis 35624 [36], although whether this is a result of a reduction of endogenous gas-forming organisms has to be speculative and an alternative explanation might be an effect on visceral sensation. 


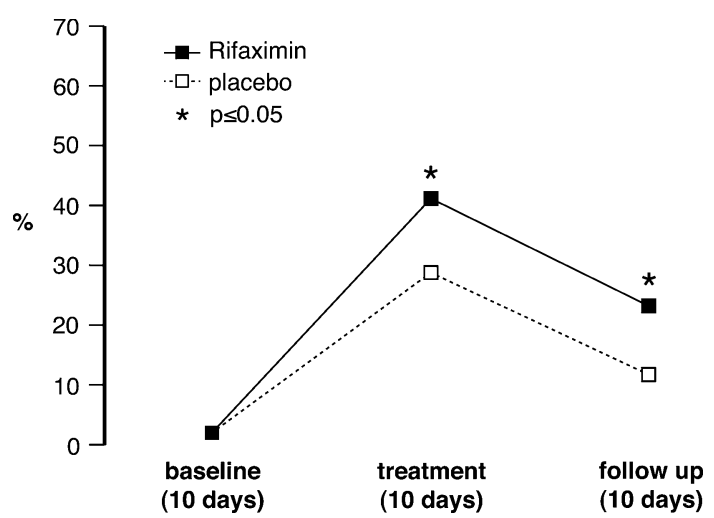

Fig. 1 A comparison of the effect of rifaximin or placebo on subjective global relief of symptoms in patients with abdominal bloating and flatulence. Taken from Ref. [22]

Visceral hypersensitivity is one of the most well described pathophysiological abnormalities in IBS [37, 38] and it has even been suggested that it might be a biological marker for the condition [39]. Consequently, it is frequently regarded as a potential target for treatment, especially by the pharmaceutical industry. The mechanism by which sensitisation is induced is not clear but it is of note that many patients date the onset of their IBS to an episode of gastroenteritis [40, 41]. It has been suggested that this could lead to sensitisation of the gastrointestinal mucosa as a result of persisting, low grade, inflammation or, alternatively, by a change in the gastrointestinal flora. It is, therefore, not surprising that it has been suggested that probiotics might have utility in reducing visceral sensitivity. To our knowledge there have, so far, been no studies of this in humans but there are promising data in animals [42-44] indicating that it is certainly worthy of further exploration.

Constipation [1] and delayed gastrointestinal transit [12] are associated with distension and it seems reasonable to suppose that improving constipation and hastening transit might lead to improvement of this problem. Consequently, it is of interest that the Barcelona group have shown that accelerating the transit of gas through the gut by administration of a prokinetic agent such as neostigmine can reduce girth [45]. Thus, if transit could be hastened by administration of a probiotic this could be an approach that might have potential in relation to reduction of abdominal distension. In physiological studies DN-173-010 has been shown to accelerate gastrointestinal transit [46-49] and, in clinical trials, to reduce the subjective reporting of bloating [37]. Therefore, this organism should, theoretically, have the potential to improve distension and this question has recently been addressed in a study using abdominal inductance plethysmography to objectively measure abdominal girth in patients with constipation-predominant IBS. Compared with a matching placebo, the active probiotic, delivered as a

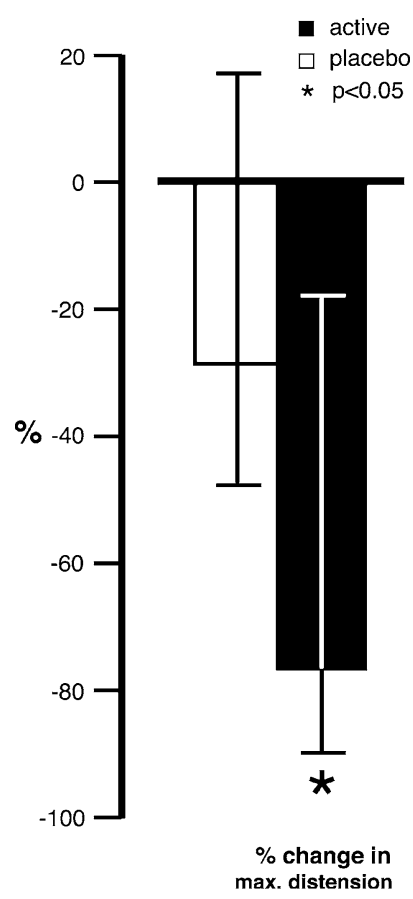

Fig. 2 A comparison of the effect of bifidobacterium lactis DN-173 010 or placebo on the percentage reduction in maximum distension (maximum distension post treatment - maximum distension at baseline/maximum distension at baseline $\times 100$ ) in patients with irritable bowel syndrome. Taken from Ref. [50]

yogurt twice daily, significantly reduced abdominal girth (Fig. 2), and this was accompanied by an acceleration in both small and large bowel transit and an improvement in symptoms [50]. It is also noteworthy that in an unrelated study, the frequency of the migrating motor complex was found to decrease in patients with IBS in whom the presence of SIBO was suggested by an abnormal lactulose breath test [51]. Interestingly, motility seemed to be enhanced once this overgrowth had been eradicated [51].

\section{Conclusion}

It seems that some probiotics and antibiotics may have a role in treating bloating and distension and this is possibly as a result of an effect on some of the mechanisms involved in the pathophysiology of these two common features of functional bowel disorders. Furthermore, it could be expected that as our understanding of the gastrointestinal microbiota and bloating and distension improves, even better control of these enigmatic symptoms might be forthcoming.

Conflict of interest Professor Whorwell has served as an advisory board member or has received research funding from the following pharmaceutical companies: Novartis Pharmaceuticals, Glaxo SmithKline, Solvay Pharmaceuticals, Rotta Research, Proctor and 
Gamble, Danone Research, Astellas Pharma, Ironwood Pharmaceuticals, Sucampo Pharmaceuticals, Almirall Pharma, Movetis UK, Norgine and Chr Hansen. Boehringer-Ingelheim, Heel GMBH.

\section{References}

1. Houghton LA, Lea R, Agrawal A, Reilly B, Whorwell PJ. Relationship of abdominal bloating to distention in irritable bowel syndrome and effect of bowel habit. Gastroenterology. 2006;131:1003-1010.

2. Agrawal A, Whorwell PJ. Review article: abdominal bloating and distension in functional gastrointestinal disorders - epidemiology and exploration of possible mechanisms. Aliment Pharmacol Ther. 2008;27:2-10.

3. Houghton LA, Whorwell PJ. Towards a better understanding of abdominal bloating and distension in functional gastrointestinal disorders. Neurogastroenterol Motil. 2005;17:500-511.

4. Serra J, Azpiroz F, Malagelada JR. Impaired transit and tolerance of intestinal gas in the irritable bowel syndrome. Gut. 2001;48:14-19.

5. Galati JS, McKee DP, Quigley EM. Response to intraluminal gas in irritable bowel syndrome. Motility versus perception. Dig Dis Sci. 1995;40:1381-1387.

6. Lewis MJ, Reilly B, Houghton LA, Whorwell PJ. Ambulatory abdominal inductance plethysmography: towards objective assessment of abdominal distension in irritable bowel syndrome. Gut. 2001;48:216-220.

7. Reilly BP, Bolton MP, Lewis MJ, Houghton LA, Whorwell PJ. A device for $24 \mathrm{~h}$ ambulatory monitoring of abdominal girth using inductive plethysmography. Physiol Meas. 2002;23: 661-670.

8. Tremolaterra F, Villoria A, Azpiroz F, Serra J, Aguade S, Malagelada JR. Impaired viscerosomatic reflexes and abdominalwall dystony associated with bloating. Gastroenterology. 2006; 130:1062-1068.

9. Villoria A, Azpiroz F, Soldevilla A, Perez F, Malagelada JR. Abdominal accommodation: a coordinated adaptation of the abdominal wall to its content. Am J Gastroenterol. 2008; 103:2807-2815.

10. Accarino A, Perez F, Azpiroz F, Quiroga S, Malagelada JR. Abdominal distention results from caudo-ventral redistribution of contents. Gastroenterology. 2009;136:1544-1551.

11. Agrawal A, Houghton LA, Lea R, Morris J, Reilly B, Whorwell PJ. Bloating and distention in irritable bowel syndrome: the role of visceral sensation. Gastroenterology. 2008;134:1882-1889.

12. Agrawal A, Houghton LA, Reilly B, Morris J, Whorwell PJ. Bloating and distension in irritable bowel syndrome: the role of gastrointestinal transit. Am J Gastroenterol. 2009;104:1998-2004.

13. Sullivan SN. A prospective study of unexplained visible abdominal bloating. N Z Med J. 1994;107:428-430.

14. Spiller RC. Postinfectious irritable bowel syndrome. Gastroenterology. 2003;124:1662-1671.

15. Madden JA, Hunter JO. A review of the role of the gut microflora in irritable bowel syndrome and the effects of probiotics. $\mathrm{Br} \mathrm{J}$ Nutr. 2002;88:S67-S72.

16. Kassinen A, Krogius-Kurikka L, Makivuokko H, Rinttila T, et al. The fecal microbiota of irritable bowel syndrome patients differs significantly from that of healthy subjects. Gastroenterology. 2007;133:24-33.

17. King TS, Elia M, Hunter JO. Abnormal colonic fermentation in irritable bowel syndrome. Lancet. 1998;352:1187-1189.

18. Whorwell PJ. Do probiotics improve symptoms in patients with irritable bowel syndrome? Therap Adv Gastroenterol. 2009;2: $37-44$.
19. Marchesi J, Shanahan F. The normal intestinal microbiota. Curr Opin Infect Dis. 2007;20:508-513.

20. Pimentel M, Chatterjee S, Chow EJ, Park S, Kong Y. Neomycin improves constipation-predominant irritable bowel syndrome in a fashion that is dependent on the presence of methane gas: subanalysis of a double-blind randomized controlled study. Dig Dis Sci. 2006;51:1297-1301.

21. Pimentel M, Park S, Mirocha J, Kane SV, Kong Y. The effect of a nonabsorbed oral antibiotic [rifaximin] on the symptoms of the irritable bowel syndrome: a randomized trial. Ann Intern Med. 2006; 145:557-563.

22. Sharara AI, Aoun E, Abdul-Baki H, Mounzer R, Sidani S, Elhajj I. A randomized double-blind placebo-controlled trial of rifaximin in patients with abdominal bloating and flatulence. Am J Gastroenterol. 2006;101:326-333.

23. Pimentel M, Lembo A, Chey WD, Zakko S, et al. Rifaximin therapy for patients with irritable bowel syndrome without constipation. N Engl J Med. 2011;364:22-32.

24. Shanahan F. Irritable bowel syndrome: shifting the focus toward the gut microbiota. Gastroenterology. 2007;133:340-342.

25. Eckburg PB, Bik EM, Bernstein CN, Purdom E, et al. Diversity of the human intestinal microbial flora. Science. 2005;308: $1635-1638$.

26. Collins S, Verdu E, Denou E, Bercik P. The role of pathogenic microbes and commensal bacteria in irritable bowel syndrome. Dig Dis. 2009;27:85-89.

27. Malinen E, Krogius-Kurikka L, Lyra A, Nikkila J et al. Association of symptoms with gastrointestinal microbiota in irritable bowel syndrome. World J Gastroenterol. 2010;16:4532-4540.

28. Malinen E, Rinttila T, Kajander K, Matto J, et al. Analysis of the fecal microbiota of irritable bowel syndrome patients and healthy controls with real-time PCR. Am J Gastroenterol. 2005;100: 373-382.

29. Matto J, Maunuksela L, Kajander K, Palva A, et al. Composition and temporal stability of gastrointestinal microbiota in irritable bowel syndrome- a longitudinal study in IBS and control subjects. FEMS Immunol Med Microbiol. 2005;43:213-222.

30. Noor SO, Ridgway K, Scovell L, Kemsley EK et al. Ulcerative colitis and irritable bowel patients exhibit distinct abnormalities of the gut microbiota. BMC Gastroenterol. 2010;10:134.

31. Krogius-Kurikka L, Lyra A, Malinen E, Aarnikunnas J, et al. Microbial community analysis reveals high level phylogenetic alterations in the overall gastrointestinal microbiota of diarrhoeapredominant irritable bowel syndrome sufferers. BMC Gastroenterol. 2009;9:95.

32. Tana C, Umesaki Y, Imaoka A, Handa T, Kanazawa M, Fukudo S. Altered profiles of intestinal microbiota and organic acids may be the origin of symptoms in irritable bowel syndrome. Neurogastroenterol Motil. 2010;22:512-519, e114-115.

33. Yang J, Lee HR, Low K, Chatterjee S, Pimentel M. Rifaximin versus other antibiotics in the primary treatment and retreatment of bacterial overgrowth in IBS. Dig Dis Sci. 2008;53: 169-174.

34. Kim HJ, Camilleri M, McKinzie S, Lempke MB, et al. A randomized controlled trial of a probiotic, VSL\#3, on gut transit and symptoms in diarrhoea-predominant irritable bowel syndrome. Aliment Pharmacol Ther. 2003;17:895-904.

35. Kim HJ, Vazquez Roque MI, Camilleri M, Stephens D, et al. A randomized controlled trial of a probiotic combination VSL\# 3 and placebo in irritable bowel syndrome with bloating. Neurogastroenterol Motil. 2005;17:687-696.

36. Whorwell PJ, Altringer L, Morel J, Bond Y, et al. Efficacy of an encapsulated probiotic Bifidobacterium infantis 35624 in women with irritable bowel syndrome. Am J Gastroenterol. 2006;101: $1581-1590$. 
37. Ritchie J. Pain from distension of the pelvic colon by inflating a balloon in the irritable colon syndrome. Gut. 1973;14:125-132.

38. Azpiroz F, Bouin M, Camilleri M, Mayer EA, et al. Mechanisms of hypersensitivity in IBS and functional disorders. Neurogastroenterol Motil. 2007;19:62-88.

39. Mertz H, Naliboff B, Munakata J, Niazi N, Mayer EA. Altered rectal perception is a biological marker of patients with irritable bowel syndrome. Gastroenterology. 1995;109:40-52.

40. Bercik P, Verdu EF, Collins SM. Is irritable bowel syndrome a low-grade inflammatory bowel disease? Gastroenterol Clin North Am. 2005;34:235-245, vi-vii.

41. Spiller R, Garsed K. Postinfectious irritable bowel syndrome. Gastroenterology. 2009;136:1979-1988.

42. McKernan DP, Fitzgerald P, Dinan TG, Cryan JF. The probiotic Bifidobacterium infantis 35624 displays visceral antinociceptive effects in the rat. Neurogastroenterol Motil. 2010;22:1029-1035, e268.

43. Ait-Belgnaoui A, Han W, Lamine F, Eutamene H, et al. Lactobacillus farciminis treatment suppresses stress induced visceral hypersensitivity: a possible action through interaction with epithelial cell cytoskeleton contraction. Gut. 2006;55:1090-1094.

44. Eutamene H, Lamine F, Chabo C, Theodorou V, et al. Synergy between Lactobacillus paracasei and its bacterial products to counteract stress-induced gut permeability and sensitivity increase in rats. $J$ Nutr. 2007;137:1901-1907.

45. Caldarella MP, Serra J, Azpiroz F, Malagelada JR. Prokinetic effects in patients with intestinal gas retention. Gastroenterology. 2002; 122:1748-1755.
46. Marteau P, Cuillerier E, Meance S, Gerhardt MF, et al. Bifidobacterium animalis strain DN-173 010 shortens the colonic transit time in healthy women: a double-blind, randomized, controlled study. Aliment Pharmacol Ther. 2002;16:587-593.

47. Meance SCC, Raimondi A. Recent advances in the use of functional foods: effects of the commercial fermented milk with Bifidobacterium animalis strain DN-173 010 and yoghurt strains on gut transit time in the elderly. Microb Ecol Health Dis. 2003;15:15-22.

48. Bouvier MMS, Bouley C. Effects of consumptionn of a milk fermented by the probiotic strain Bifidobacterium animalis DN-173 010 on colonic transit times in healthy humans. Bioscience Microflora. 2001;20:43-48.

49. Meance SCC, Turchet P. A fermented milk with a bifidobacterium probiotic strain DN-173 010 shortened oro-fecal gut transit time in elderly. Microb Ecol Health Dis. 2001;13:217-222.

50. Agrawal A, Houghton LA, Morris J, Reilly B, et al. Clinical trial: the effects of a fermented milk product containing Bifidobacterium lactis DN-173-010 on abdominal distension and gastrointestinal transit in irritable bowel syndrome with constipation. Aliment Pharmacol Ther. 2009;29:104-114.

51. Pimentel M, Soffer EE, Chow EJ, Kong Y, Lin HC. Lower frequency of MMC is found in IBS subjects with abnormal lactulose breath test, suggesting bacterial overgrowth. Dig Dis Sci. 2002;47:2639-2643. 